\title{
Different methods for ethical analysis in health technology assessment: An empirical study
}

\author{
Samuli I. Saarni \\ National Institute for Health and Welfare; the Finnish Office for Health Technology \\ Assessment; and University of Helsinki and Helsinki University Central Hospital
}

Annette Braunack-Mayer

University of Adelaide

\section{Bjørn Hofmann}

University College of Gjøvik; Norwegian Knowledge Center for the Health Services; and University of Oslo

\section{Gert Jan van der Wilt \\ Radboud University Nijmegen Medical Centre}

Objectives: Ethical analysis can highlight important ethical issues related to implementing a technology, values inherent in the technology itself, and value-decisions underlying the health technology assessment (HTA) process. Ethical analysis is a well-acknowledged part of HTA, yet seldom included in practice. One reason for this is lack of knowledge about the properties and differences between the methods available. This study compares different methods for ethical analysis within HTA.

Methods: Ethical issues related to bariatric (obesity) surgery were independently evaluated using axiological, casuist, principlist, and EUnetHTA models for ethical analysis within HTA. The methods and results are presented and compared.

Results: Despite varying theoretical underpinnings and practical approaches, the four methods identified similar themes: personal responsibility, self-infliction, discrimination, justice, public funding, and stakeholder involvement. The axiological and EUnetHTA models identified a wider range of arguments, whereas casuistry and principlism concentrated more on analyzing a narrower set of arguments deemed more important. Conclusions: Different methods can be successfully used for conducting ethical analysis within HTA. Although our study does not show that different methods in ethics always produce similar results, it supports the view that different methods of ethics can yield relevantly similar results. This suggests that the key conclusions of ethical analyses within

\footnotetext{
The contribution of Heidi Anttila, Suoma E. Saarni, Pertti Mustajoki, Vesa Koivukangas, Tuija S. Ikonen, and Antti Malmivaara for the ethical analysis of FinOHTA HTA on bariatric surgery is gratefully acknowledged. Each of the author has contributed to the conception, design, and writing of the study, with Dr. Saarni drafting the final version which each author has approved. The contents have not been published or are not being considered for publication elsewhere, with the exception of the full HTA conducted by FinOHTA on which the EUnetHTA parts of the study are based and which has been published in Finnish (as clearly stated and acknowledged in the manuscript).
} 
Saarni et al.

HTA can be transferable between methods and countries. The systematic and transparent use of some method of ethics appears more important than the choice of the exact method.

Keywords: Health technology assessment, Ethics, Axiology, Casuistry, Principlism, EUnetHTA

Ethical analysis has long been acknowledged as an essential part of HTA:

Technology assessment is a comprehensive form of policy research that examines the short- and long-term social consequences (e.g., societal, economic, ethical, legal) of the application or use of technology (19).

There are good theoretical grounds for including ethics in health technology assessment (HTA). Technologies and HTAs are not executed in a vacuum, but always in a social and value context. The inclusion of ethical analysis can help take this into account and improve the usefulness of HTA for several reasons $(2 ; 7 ; 9 ; 12 ; 24 ; 25)$. Implementing new technologies in health care can have morally relevant consequences. Technologies carry with them values that can challenge the current mores and attitudes of society. Every HTA requires many value-decisions to be made during the assessment process. Finally, the whole HTA can be seen as a value-laden enterprise, as it usually aims to improve health care and healthcare decision making $(7 ; 9 ; 12 ; 24 ; 25)$. These considerations show that the role of ethics in HTA is not limited to the analysis of consequences of implementation and improvements in health decision making. Ethics can also investigate the meanings of technology and explicate and justify the normative structure of the HTA itself.

In practice, however, ethical analysis is still not included in the vast majority of HTAs $(2 ; 9 ; 12 ; 17)$. One of the rationales underpinning this study is that one reason for this is the lack of knowledge about the available methods and, especially, their relative properties.

There is no lack of methods in ethics. Quite the contrary, there is a plurality of methods in use which are enthusiastically supported by their developers and users. There is, however, a lack of consensus about which methods are best. More importantly, there is a lack of consensus about why and how much it matters which method one uses for ethical analysis. For example, the recent EUnetHTA project presented ten different methods used for ethical analysis within HTA, but only vaguely recommended using the one that best suits the topic, local culture and HTA organization $(15 ; 23)$. In this study, we suggest that understanding the practical differences and similarities between methods and then choosing the most appropriate for the context is more important than trying to agree on one theoretically superior method for all instances.

The study aims to (i) present, compare, and contrast four different methods of ethical analysis in HTA; and (ii) compare results and highlight strengths and weaknesses of these methods.

The study aims to offer practical suggestions for those planning to include ethical analyses in their HTAs and help in choosing the most suitable method.

\section{METHODS}

Surgical treatment of obesity is often believed to be controversial by many people including professionals (18). The causes of obesity are multifactorial. Obesity surgery is the most effective treatment of morbid obesity, but does not remove the social causes of obesity nor solve the "obesity epidemic" on a public health level $(4 ; 5)$. The number of operations performed has increased dramatically, but the number of obese people potentially benefiting from surgical treatment outnumbers the capacity and currently available resources in many countries. Obesity is socioeconomically unequally divided, and the obese are discriminated against in many ways (20), but it is unclear how increasing bariatric surgery affects this.

A full HTA on bariatric surgery was conducted by FinOHTA years 2008-2009, using the EunetHTA core model and including an analysis of the ethical (led by SIS), social, legal and organizational domains. For comparison, ethical issues in bariatric surgery were independently considered using three different methods by different researchers with experience on these methods: axiology (B.H.), casuistry (G.J.V.W.), and principlism (A.B.-M.). For these ethical analyses, which were not part of full HTA, the literature searches on effectiveness of bariatric surgery conducted by FinOHTA were available, but not the results of the FinOHTA which were published so far only in Finnish.

Below, we briefly describe the four methods of ethical analysis applied. Using bariatric surgery as an example, we describe and compare the application and results of these methods.

\section{EUnetHTA Model}

The EUnetHTA project did not focus on creating a new methodology, but on standardization of available methods and increasing international transferability of HTAs $(16 ; 23)$. In contrast to most previous HTA methodology manuals, however, EUnetHTA presents a practical way to integrate ethical considerations into the whole HTA process.

The EUnetHTA approach emphasizes the value-ladeness of HTA by considering ethical issues already in the planning 
phase of the HTA. Many issues that are decided in the planning phase such as topic selection, outcome variables, study methods (e.g., need for separate social, organizational, ethical analyses) are ethically relevant and define the limits of the HTA. Ethical issues are then identified using a questionbased format, adapted from axiology (8), and addressed in a reflective process between those responsible for ethical analysis and the other domains of assessment. No single method of balancing ethical issues is stipulated, but several methods are presented for the user to choose from. Wherever possible, ethical analysis is conducted in close collaboration with content experts. Reporting the conclusions is ideally done in a structured format, to enhance both transparency of the argumentation and international transferability. The whole model (15) is available at www.eunethta.net.

\section{Casuistry}

A common feature of all variants of casuistry is reliance on comparing cases in the resolution of a specific moral dilemma. Reasoning is based on paradigms and analogies, and moral norms are not considered universal; rather they hold with certainty only in typical conditions and circumstances (14). This study is based on the model proposed by Brennan (3).

The key task in this type of inquiry is to propose cases that bear sufficient similarity to the case under investigation, which are morally less confusing or contentious. A comparison of the various cases should reveal whether the same sort of moral inferences may be made in each case. This should help to reveal which moral principle seems to be at stake, and what practical implications follow from it. This part of the inquiry is called the explication of the moral principle (3). Ideally, it is conducted by a small (typically up to six) group of individuals with complementary expertise and experience (e.g., moral, medical, and experiential). When it remains unclear to the participants whether the selected cases bear sufficient resemblance to the case under investigation, they may take recourse to the rationale of a moral principle. The rationale provides an answer to the question why the moral principle is important in the first place, and can act as controlling norm on its correct explication (3). Moral expertise consists of a broad knowledge of potentially relevant cases (repertoire), and the ability to relate specific cases with abstract principles and their rationales and to identify relevant differences and commonalities between cases. The key reason for opting for this type of inquiry is the view that moral judgments cannot be derived deductively from moral principles (14).

\section{Axiology}

Axiology emphasizes the insight that science and technology is a social activity governed by norms and values of various kinds. As health technology is applied in a social setting where there is interplay of many different kinds of norms and values, HTA should highlight and address the norms and values involved in the implementation and use of a health technology.

The axiological approach consists in six steps (10).

(i) Identify and analyze the moral challenges that are typical for the health technology; (ii) Identify stakeholders; (iii) Select a set of morally relevant questions by selecting from a list of thirty-two questions $(8 ; 9)$, which highlight value issues in regard to the implementation of health technologyJustify the selection; (iv) Perform literature search on the basis of the steps i-iii; (v) Analyze the selected questions (in step 3) on the basis of the literature search (step iv), hearings with stakeholders, and results from qualitative research; and (vi) Summarize the analysis and highlight the most important value issues.

The aim with addressing norms and values through the set of morally relevant questions is to provide an open, transparent, and informed decision making process.

\section{Principlism}

Principlism has become a very popular framework in bioethics, the most quoted formulation being given by Beauchamp and Childress (1). Principlists propose that the analysis of ethical issues in biomedicine can be conducted with recourse to four moral principles: respect for autonomy (with a focus on human free will and agency); beneficence (the obligation to do good for others); non-malfeasance (the minimization of harm to others); and justice (the distribution of benefits and burdens). These principles are used to analyze and resolve ethical issues in a coherentist manner, which is "a matter of the mutual support of many considerations, of everything fitting together into one coherent whole"(1). In practice, the principles are usually applied to the ethical issue or case under discussion in turn; once the implications of each principle are clear, actual obligations to act in certain ways are determined by balancing and weighing all norms against each other, needing special reasons to infringe any of the principles or giving priority to any norms.

\section{RESULTS}

We present here a concise overview of the process and results of the assessments; the more extensive analyses using casuistry, axiology, and principlism are included as Supplementary Tables 1, 2, and 3, respectively, which can be viewed online at www.journals.cambridge.org/thc2011022. EUnetHTA results will be published separately (22). The analyses concern only the ethical aspects, and they are based on a consensus about the effectiveness and safety of bariatric surgery in reducing weight and several conditions associated with obesity.

\section{EUnetHTA model}

This section is based on the FinOHTA HTA on obesity (bariatric) surgery which used the EUnetHTA model (13). 
Discussions about ethical issues in the planning phase influenced the HTA in several ways: A strict PICO (patientintervention-control-outcome) definition was considered too narrow, as it was anticipated that the most significant ethical problems would not relate to the surgical procedure as such, but to obesity and its treatments in general. Internists and general practitioners were included in the expert group in addition to surgeons. The endpoints of analysis were expanded to include as many patient-relevant outcomes as possible. A systematic review of both quantitative and qualitative literature of psychosocial outcomes of bariatric surgery was commissioned. Perhaps the single most important insight encouraging full ethical, social, and organizational analysis was that the planning group unanimously agreed, after a preliminary literature search, that (i) bariatric surgery appears uncontroversially effective and (ii) a thorough HTA is definitely needed. A survey of Finnish hospitals was conducted to evaluate their views of the demand and possible expansion of bariatric surgery volumes.

The work on answering the questions included in the core model was done in a reflective manner: first, a discussion about the relevance of the issue, then clarification of the issue and information needs it entailed. Literature searches were conducted in a relatively late phase based on the themes identified on the discussions, using existing guidelines (6). Finally, the ethicist (SIS) combined the discussions around the questions into larger themes.

Two main themes emerged: value-questions relating to obesity and obesity treatments, and ethical evaluation of the consequences of expanding the provision of bariatric surgery. The key arguments presented were as follows:

The surgical operation as such does not pose major new ethical dilemmas, but the main ethical problems relate to obesity and obesity treatments in general. Attitudes toward obesity-especially etiology, ideal treatments, and preventive methods-determine the attitudes toward bariatric surgery. If obesity is considered to be caused by free and reversible individual choices, it can be questioned why publicly financed surgical treatment should be offered? The EUnetHTA analysis suggests that the role of individual responsibility for obesity, and therefore for the need of surgery itself, is fairly limited. This suggests that the question of whether "obesity" is a "disease" is not relevant, and it is appropriate to treat the condition in the same way as other medical conditions. Furthermore, the individual and social consequences of medicalizing obesity are potentially significant but were found difficult to predict and probably varying by stakeholder.

There are several important moral consequences of doing bariatric surgery. Actively supporting patient autonomy is justified, as most operations are permanent and require major changes in eating habits to be effective; the patient must be thoroughly aware of these issues. Justice considerations are also significant, as obesity is more common in people of lower socioeconomic status who may fail to receive treatment for a variety of reasons if not actively encouraged. The need for explicit rationing of operations is also important, as it is unlikely resources will be available to operate on all who could benefit from the treatment. The operation itself is only part of a process of surgical treatment of obesity, usually preceded by years of dieting, and followed by life-long follow-up. Rationing should thus be about whole process of the treatment of obesity, and not just bariatric operations. Finally, balancing the different arguments from the viewpoints of different stakeholders is needed.

\section{Casuistry}

Bariatric surgery has attracted considerable public attention. Despite it being an effective treatment, its acceptance for public funding has been disputed. It has been asked, for example, why we ration many practical health-aids but cover the costs of "self-inflicted" conditions such as obesity. Thus the main ethical problem - the moral perplexity - seems to relate to public funding of bariatric surgery. Should obese people start taking responsibility for their health, and is it fair to require public funding for obesity treatments? The moral hypothesis to be considered, therefore, is that it is morally wrong that a society should bear the costs of treatment for conditions for which individuals can be held responsible. Casuistic inquiry then proceeds by searching for relevant paradigmatic cases, and by establishing commonalities and differences between those and the case under investigation.

A Drowning Accident. Should we stop and help a person who is about to drown, if we can do that without risk to ourselves, even if that person has knowingly taken a huge risk? Most of us would probably answer yes, which erodes the credibility of the claim that people with morbid obesity should not be helped. However, drowning is an acutely lifethreatening incident, so the inference is questionable.

Burnout. Should we try to help a colleague with burnout, also when he has been repeatedly warned to better manage his workload? Again, most of us would probably say yes. However, in this case the person is a known colleague, which might bring special obligations.

Attempted Suicide. Should we treat a person who has taken a large, although not definitely lethal, dose of medications? Or should we refrain, as the act is self-inflicted? Here, again, we would be hard-pushed to leave the victim to his own resources.

Other cases could be put forth (e.g., medical care for people who sustained sports injuries) but, so far, little support has been gained for the initial hypothesis. This suggests that excluding bariatric surgery from public coverage because the condition is self-inflicted would require us to change our concept of individual responsibility. This, in turn, would require a new justification for continued coverage of many services currently covered (e.g., for people with sports injuries, sexually transmitted diseases, or lung cancer). 
When we are still in doubt, we may turn to the rationale for the moral concept: what is it that we wish to uphold by holding people individually responsible? One view is that by holding people responsible we aim to establish or reinforce particular moral relationships with them, to confirm our mutual commitment to similar values or principles. So, what values are we trying to protect by holding individuals responsible for being obese? One possible answer is that people should take care of their health, minimize the burden on others and health care, and resist excess and idleness. Interrogating the rationale for the moral concept aims to show us what we are really seeking when we try to fit individual responsibility to bariatric surgery. If we locate this goal we can better assess what really is the most effective way to achieve it. For example, if the rationale is to decrease the burden on the public health system, excluding or reducing bariatric surgery might be counterproductive. In summary, then, the inquiry failed to support the hypothesis that covering the costs of bariatric surgery is morally problematic.

The casuistic analysis does not purport to be conclusive. People are free to bring up other cases; indeed they might frame the problem differently from the outset (formulating a moral hypothesis related to distributive justice), or they might provide different rationales. The point is: casuistic analysis provides structure, it helps to explore the nature of our moral concerns, it enables us to be slightly more consistent in our judgments, and it makes normative positions explicit that could otherwise easily have remained implicit.

\section{Axiology}

In this section, we present the value-questions in step 3 of the method presented above and the most relevant themes analyzed in step 5. A more elaborate analysis can be found in (11).

What Are the Morally Relevant Consequences of Bariatric Surgery?. Analysis of morally relevant consequences naturally hinges on the empirical data: how effective bariatric surgery is, and what is the quality of the data. Important consequences to consider are at least: reduced mortality and morbidity, increased quality of life, positive psychological effects, economic impacts, and risks of the procedure.

Is Personal Autonomy Challenged?. If crucial information on effectiveness and safety is not available, it is impossible to obtain valid informed consent from potential patients. Psychiatric co-morbidity may reduce competence to consent. On the other hand, assessment of reduced competence may result from prejudices. Mandatory preoperative dietary counseling reduces choice and challenges autonomy.

Is Human Integrity or Dignity Challenged? Does Bariatric Surgery Change Our Conceptions of Certain Persons?. Substantial weight discrimination has been documented in many countries, and widespread nega- tive stereotypes exist even among health care professionals, so integrity and dignity might be at risk. Whether bariatric surgery is a solution to this social problem is a pertinent question.

Does Bariatric Surgery Challenge or Change Social Conceptions or Arrangements?. Food, eating and relationship to the body are both strongly cultural and very private phenomena, making interventions against obesity important interventions into people's life. Bariatric surgery supports social condemnation of fat and, as obesity is often considered self-inflicted, might increase the blame on and feelings of guilt among obese people. However, it is difficult to defend not including bariatric surgery in publicly financed health care for suffering persons.

How Does Bariatric Surgery Relate to Challenges to Modern Medicine?. Bariatric surgery may be viewed as part of the medicalization of the modern life-world, related to moral, political, or economic incentives to transform various problems into medical ones.

Does Bariatric Surgery Challenge Basic Conceptions in Health Care?. The definition of obesity is not strict and has changed over time (11). It is debated whether obesity is a disease, and how normative the concept is. Acceptance of bariatric surgery can influence this debate and alter our conception of disease.

Does Bariatric Surgery Challenge or Change the Relationship Between Physician and Patient? Physicians appear to have the same prejudice as the general public when it comes to obesity.

How Does Bariatric Surgery Affect the Distribution of Health Care?. Bariatric surgery is costly and effective so it might lead to re-allocation of resources, especially as only a minority of eligible persons is offered bariatric surgery. Obesity affects disadvantaged minorities disproportionately, and so does the distribution of bariatric surgery.

What Are the Interests of the Surgeons?. Surgeons might gain from bariatric surgery and the medicalization of obesity, making their role in promoting bariatric operations problematic. On the other hand, prejudices among physicians might decrease the availability of operations.

What Is the Status or Symbolic Value of Bariatric Surgery?. Obesity is a disease with low prestige within health care, and its status as a disease is sometimes questioned. However, "life-style diseases" are increasingly the focus of public and political attention.

Is the Selection of End-points Morally Relevant?. It is morally relevant whether the end-point of assessment is weight, sustained weight reduction, health-related quality of life, reduced morbidity, increased life length, adverse effects, reduced psychiatric distress, increased self-esteem, or costs. 
In conclusion, there are challenges with autonomy and informed consent, due to complex information, psychiatric co-morbidity, and voluntariness. Obesity is subject to prejudice and discrimination, and there is unjust access to bariatric surgery. Lack of access to bariatric surgery, but also pressure to undergo bariatric surgery, may enhance prejudice and discrimination. Bariatric surgery can be seen from many perspectives: as a preventive intervention with regard to serious health problems, as a method for improving the wellbeing of a vulnerable group of persons, as a symptom-oriented intervention in people's life-world, as a mere confirmation and enhancement of the cultural and social prejudices and discriminations being at the core of the problem. Such ethical issues should be addressed in the decision making process for bariatric surgery.

\section{Principlism}

Bariatric surgery raises a wide range of ethical questions, related to consent (21), conflicts of interest (18), and the balance of harms and goods. However, it is the fact that obesity is considered a "lifestyle" problem that makes bariatric surgery particularly ethically challenging. Thus, we concentrate on the question of whether bariatric surgery should be publicly funded in the same way as treatments for conditions that seem completely outside of individual control.

A principlist approaches the question by first identifying which of the four key moral principles are pertinent to this case. The most immediately relevant principle is that of justice or fairness. Justice requires that like cases be treated in like manner. But what makes candidates for bariatric surgery like or unlike other people? Four criteria for just decisions are commonly considered and are described briefly below: need, capacity to benefit, rights and merit. Do candidates for bariatric surgery differ in these respects from other patients? If they do not, there are no grounds for discriminating against them.

Allocating according to clinical need is a guiding principle for most health care systems. We usually think that diabetes, hypertension and sleep apnea-and even their significant risk-indicate clinical need. As obesity is a risk factor for these conditions it should indicate similar need; cases of mild obesity or operations for cosmetic reasons alone can be questionable.

Considering capacity to benefit is mostly an empirical question. The literature suggests bariatric surgery is effective, even cost-effective, and can benefit certain patients comparably to many established procedures.

The weight of equal rights considerations varies, especially in comparison to capacity to benefit, between health care systems. Regardless of the differences, the key issue here is not whether we have a right to publicly funded bariatric surgery at all but whether all people in a particular country or community have the same right. In many countries, the argument about right to bariatric surgery quickly becomes a question of individual responsibility and past conduct; thus it becomes a question of merit.

Merit suggests that past contribution, effort or status is important in health care decisions. This makes sense if people can really influence their merits. Unfortunately, the link between surroundings, character, actions, and free will is very complicated in general, and in the case of obesity in particular. Obesity depends partly on genetics, childhood experiences, socio-economic status, and society, so attributing full responsibility to individuals is problematic. Still, attributing some responsibility in most cases seems reasonable, so the merit criterion gives some grounds for limiting bariatric surgery.

In conclusion, a principlist analysis which focuses on the principle of justice suggests that, on criteria based on clinical need and capacity to benefit, bariatric surgery should be provided, but emphasizing rights and merit may lead to a different conclusion. Because our conclusions based on merit and rights are more tentative, the balance of argument favors providing public funding for bariatric surgery.

\section{DISCUSSION}

We have presented and used four different methods for analyzing ethical issues in HTA. Our aim has been to present the relative properties of the methods, rather than to find the best method.

In this case study, all four methods have identified and analyzed similar themes: in different ways they address questions related to personal responsibility, self-infliction, discrimination, and justice. EUnetHTA and axiology, with related processes, identify a wide range of arguments. Principlism and casuistry, in particular, draw our attention to the question of personal responsibility for obesity. All methods also consider the question of whether bariatric surgery should be publicly funded.

All methods arrive at similar conclusions regarding responsibility: each concludes that it is difficult to sustain the argument that obesity is self-inflicted in the sense that being obese is something over which individuals have complete control. The principlist analysis has some reservations about this conclusion, but suggests that arguments from capacity to benefit and need outweigh these reservations. On that basis, all four methods appear to conclude that bariatric surgery should be funded in the same way as other surgical treatments.

There are also similarities in the ways in which each method is used. All methods take stakeholders into account. They use systematized knowledge as inputs to the analysis, and contain an analysis component. Not surprisingly, though, there are also important differences between the methods in the ways in which the analyses are conducted. One obvious difference relates to their roots: the question-based methods (axiological and EUnetHTA) require wide consideration of many value-related aspects of the technology. On the other 
hand, the methods based on ethical theory (casuistry and principlism) allow one to concentrate on a thorough analysis of the topic or theme considered most important. (Whether principlism is an ethical theory is controversial. However, for the sake of this analysis it can be seen as a constantly evolving reflective equilibrium with some basis in common morality as a framework.) This reflects the fact that the approaches work at somewhat different theoretical levels: casuistry and principlism can also be used as methods for answering the questions identified by the axiological / EUnetHTA frameworks. In other words, the approaches can be used together: the question-based approaches can be used to identify the ethical issues, and casuistry, principlism, or other mid-level theories can be used to deepen the ethical analysis on each issue, or to appraise which issues are the most relevant for the technology under study.

Given the above factors, principlist and casuist analyses may be easier to conduct as separate add-on ethical analyses provided by ethicists, or used in situations where the ethically problematic themes requiring clarification are identified. Axiological and EUnetHTA analyses may be more appropriately integrated into the HTA process, or used in situations where the ethically problematic issues are not readily identified.

The main weakness of this study is that the analyses are not perfectly comparable, as only one was done as part of a full HTA on the topic. Thus, the other analyses were not integrated into a HTA process, but were done as a separate exercise, without the usual stakeholder involvement, and must thus be seen as more tentative. This problem is difficult to overcome, as conducting several full HTAs on the same topic in different countries is not yet common, and may not be feasible.

An interesting question our study cannot answer is how culture, ethical method, and the individual ethicist interact in the process and results of ethical analysis in HTA. Other ethicists may possibly arrive at different results applying the same methods used in this study. However, we believe these would be differences in emphasis and selection of topics, and that alternative ways of applying the methods would be commensurable. Ethical analysis performed in one (western, developed, public health care) country is likely to be relevant for other similar countries.

\section{CONCLUSIONS}

Our study supports the view that different methods can be successfully and systematically used for conducting ethical analysis within HTA. In this case, the four methods used yielded relatively similar results. This does not prove that different methods of ethics converge. Still, our results do not support the view that different methods are likely to yield significantly different results, and that results of ethical analysis in HTA will not be transferable enough. More studies and theoretical work are needed.
At this state of the art, it seems one can justifiably choose among several methods of ethics when conducting an ethical analysis within HTA. The systematic use of some method of ethics makes the analysis more reproducible and transparent and increases accountability. There is no need to wait for a consensus on methods of ethics among philosophers. It may never come and waiting would hamper the development and use of HTA.

\section{SUPPLEMENTARY MATERIAL}

Supplementary Table 1

Supplementary Table 2

Supplementary Table 3

www.journals.cambridge.org/thc2011022

\section{CONTACT INFORMATION}

Samuli I. Saarni, MD, MSocSc, PhD (Samuli.saarni@ helsinki.fi), National Institute for Health and Welfare, Departments of Mental Health and Substance Abuse Services and the Finnish Office for Health Technology Assessment, P.O. Box 30, 00271 Helsinki, and University of Helsinki and Helsinki University Central Hospital, Department of Psychiatry, Helsinki, Finland

Annette Braunack-Mayer, PhD, BMedSci(Hons) (annette. braunackmayer@adelaide.edu.au), Head, School of Population Health and Clinical Practice, University of Adelaide, Level 11, Terrace Towers, 178 North Terrace, Mail Drop Box DX 650 207, Australia, SA 5005

Bjørn Hofmann, MSc, PhD (b.m.hofmann@medisin. uio.no), University College of Gjøvik, P.O. Box 1, N-2802 Gjøvik, Norway; Norwegian Knowledge Center for the Health Services, P.O. Box 7004, St. Olavs plass, N-0130 Oslo, Norway; and University of Oslo, P.O. Box 1130, Blindern, N-0318 Oslo, Norway

Gert Jan van der Wilt, MSc, PhD (G.vanderWilt@ebh. umcn.nl), Department of Epidemiology, Biostatistics and HTA (133), Radboud University Medical Centre, P.O. Box 9101, 6500 HB Nijmegen, The Netherlands

\section{CONFLICT OF INTEREST}

Samuli Saarni has received travel funding from AstraZeneca. The other authors report they have no potential conflicts of interest.

\section{REFERENCES}

1. Beauchamp T, Childress J. Principles of biomedical ethics. 6th Edition (1 st ed. 1979). New York: Oxford University Press; 2008.

2. Braunack-Mayer AJ. Ethics and health technology assessment: Handmaiden and/or critic? Int J Technol Assess Health Care. 2006;22:307-312. 
3. Brennan J. The open-texture of moral concepts. London: Macmillan; 1977.

4. Buchwald $\mathrm{H}$, Avidor $\mathrm{Y}$, Braunwald E, et al. Bariatric surgery: A systematic review and meta-analysis. JAMA. 2004;292:17241737.

5. Colquitt JL, Picot J, Loveman E, Clegg AJ. Surgery for obesity. Cochrane Database Syst Rev. 2009:CD003641.

6. Droste S, Gerhardus A, Kollek R. Methoden zur Erfassung ethischer Aspekte und gesellschaftlicher Wertvorstellungen in Kurz-HTA-Berichten - eine internationale Bestandsaufnahme. [Methods for the assessment of ethical aspects and moral concepts in society in health technology assessment reports - an international survey]. Schriftenreihe HTA des DIMDI vol. 9. Köln; 2003.

7. Heitman E. Ethical issues in technology assessment. Conceptual categories and procedural considerations. Int J Technol Assess Health Care. 1998;14:544-566.

8. Hofmann B. Toward a procedure for integrating moral issues in health technology assessment. Int J Technol Assess Health Care. 2005;21:312-318.

9. Hofmann B. On value-judgments and ethics in health technology assessment. Poiesis Prax. 2005;3:277-295.

10. Hofmann B. Etikk $i$ vurdering av helsetiltak. Utvikling av en metode for å synliggjøre etiske utfordringer ved vurdering av helsetiltak. [In Norwegian with an English summary: Ethics in the assessment of health technology]. Oslo: Norwegian Knowledge Center for the Health Services; 2008.

11. Hofmann B. Stuck in the middle: The many moral challenges with bariatric surgery. Am J Bioeth. 2010;10:3-11.

12. Hofmann BM. Why ethics should be part of health technology assessment. Int J Technol Assess Health Care. 2008;24:423429.

13. Ikonen T, Anttila H, Gylling H, et al. Sairaalloisen lihavuuden leikkaushoito. [Surgical treatment of morbid obesity. In Finnish]. Helsinki: National Institute for Health and Welfare; 2009.
14. Jonsen AR. Casuistry as methodology in clinical ethics. Theor Med. 1991;12:295-307.

15. Lampe K, Mäkelä M, eds. HTA Core Model for medical and surgical interventions 1.0R: http://www.eunethta.net/upload/WP4/ Final\%20Deliverables/HTA\%20Core\%20Model\%20for\% 20Medical\%20and\%20Surgical\%20Interventions $\% 201 \% 200$ r. pdf 2008 (accessed August 29, 2011).

16. Lampe K, Mäkelä M, Garrido MV, et al. The HTA core model: A novel method for producing and reporting health technology assessments. Int J Technol Assess Health Care. 2009;25(Suppl 2):9-20.

17. Lehoux P, Tailliez S, Denis JL, Hivon M. Redefining health technology assessment in Canada: Diversification of products and contextualization of findings. Int J Technol Assess Health Care. 2004;20:325-336.

18. Mitka M. Surgery for obesity: Demand soars amid scientific, ethical questions. JAMA. 2003;289:1761-1762.

19. Office of Technology Assessment. Development of medical technology: Opportunities for assessment. Washington, DC: Office of Technology Assessment; 1976.

20. Puhl RM, Heuer CA. The stigma of obesity: A review and update. Obesity (Silver Spring). 2009;17:941964.

21. Raper SE, Sarwer DB. Informed consent issues in the conduct of bariatric surgery. Surg Obes Relat Dis. 2008;4:60-68.

22. Saarni SI, Anttila H, Saarni SE, et al. Ethical issues of bariatric surgery - a health technology assessment. Obes Surg. 2011 [Epub ahead of print].

23. Saarni SI, Hofmann B, Lampe K, et al. Ethical analysis to improve decision-making on health technologies. Bull World Health Organ. 2008;86:617-623.

24. ten Have H. Ethical perspectives on health technology assessment. Int J Technol Assess Health Care. 2004;20:71-76.

25. Van der Wilt GJ, Reuzel R, Banta HD. The ethics of assessing health technologies. Theor Med Bioeth. 2000;21:103115. 